\title{
Development of Virtual Pulse Simulation for Pulse Diagnosis Studies
}

\author{
https://doi.org/10.3991/ijim.v12i7.9640 \\ Moragot Kandee ( $\left.{ }^{\bowtie}\right)$, Poonpong Boonbrahm \\ Walailak University, Nakhon Si Thammarat, Thailand \\ morakhot 2546 @hotmail.com \\ Valla Tantayotai \\ Thai Association of Diabetes Educators, Bangkok, Thailand
}

\begin{abstract}
Pulse signals can be used to observe the early sign of patients' health problems. From medical researches, monitoring the characteristic of arterial pulse waveform shows some risk indicator of specific diseases, e.g., hypertension, cardiovascular and heart failure diseases. A simple way to get arterial pulse wave is by using fingers to touch the radial artery position on the wrist. In the traditional Chinese medicine, a physician can use the information of arterial pulse wave-form to identify diseases based on the physician's ability and experience. The improvement of the skill in pulse measurement can be improved by training using various kinds of pulses that represent each disease. This paper proposes a development of the virtual pulse simulation using Augmented Reality (AR) and haptic device for pulse diagnosis studies under various situations. The pulse simulation generates arterial pulse waveforms based on Sine and Gaussian functions. In this study, the mathematical model can generate the pulse wave like human pulse by setting up specific parameters. We can generate pulse waveform which representing different kinds and states of diseases by varying the mathematical model and parameters such as pulse rate or pulse pressure. The features of this work include how to generate force feedback from the mathematical models using the haptic device and how the virtual 3D can display visual feedback. The pulse simulation is useful for the health sciences students, especially the nursing students in training to identify some diseases. The evaluation of the system was carried out by first-year nursing students regarding usability, satisfaction, and performance.
\end{abstract}

Keywords - haptic device, virtual pulse simulation, pulse diagnosis study, pulse waveform modeling

\section{Introduction}

A study of pulse measurement is a basic learning for medical and nursing students because the pulse is a vital sign that can use to identify the condition of the vascular system and patients' symptoms. When the students have experience and skills in the pulse measurement, they can use diagnose some diseases faster and use pulse data for 
making a treatment decision properly. For above reasons the training of pulse measurement is needed to improve the skill of nursing students. In general, the nursing students practice radial pulse measurement with their friends by using their fingers in examinations. By that way, they can only experience the regular pulse (such as heart rate, rhythm, and amplitude). The students hardly have experience in examining the irregular pulse. The pulse simulation can provide the various situations of pulses and unlimited repetitions for the training.

A new kind of the training development in education and learning is a haptic application. In the research survey, the haptic technology has been used as a medical training tool for the interaction between users [1]. During the training, the haptic device responds force feedback to the users which is the process of human-computer interaction for improving their touch sensation. By using Augmented Reality (AR) technology in the training, it will increases a more realistic learning experience and fully controllable environments in the pulse simulation which can make the students feel the pulse and practice with the various cases of the pulse.

The objective of this study is to develop the virtual pulse simulation using Augmented Reality and haptic device for pulse diagnosis studies under various situations. This work is aimed to find the way to use the pulse diagnosis training of radial artery pulse waveforms to train the nursing students on touch and cognitive skills.

\section{$2 \quad$ Literature Review}

A review of virtual simulations in medical education, the haptic device can be applied as a learning tool on the different task of taking pulse and palpation training in medicine [2]. For examples of the related works, Ullrich et al [3] used the haptic to simulate the brachial pulse of human and created blood flow in virtual palpation simulation. They developed the force feedback of rectangular pulse wave in normal pulse case and modified palpation interaction method of two haptic devices representing in [4]. Coles et al [5] simulated square and triangular waveforms of the femoral pulse in a virtual environment of interventional radiology using the haptic palpation simulation. LeClair et al [6] also presented rectangle, triangular, and physiologically-based waveforms of cardiovascular disease using haptic interface. All of above researches proposed the virtual reality simulations in different pulse generating and used the different haptic devices (such as Phantom Omni [3-4] and Novint Falcon [5-6]) for response force feedback. Some parts of the haptic device had been modified to support the pulse palpation of normal cases [4-5].

Some researches used the pulse waveform generating without virtual and haptic interface. The arterial pulse waveforms was created by the optimum modeling of carotid and radial pulse using Gaussian function in [7]. In addition, the information of artery wave changes in healthy and cardiovascular disease volunteers was used to normalize by the Gaussian fitting method [8]. Zhu et al [9] also used Gaussian function to deduce the equation for radial waveform of patients' signals. The previous studies of the pulse waveforms were generated by Gaussian functions and can used to indicate some diseases such as hypertension, cardiovascular disease, and heart failure diseases [10]. 
In this research, we develop the pulse simulation by using AR and Phantom Omni for the training of radial artery pulse under various situations. AR technology can display the virtual 3D model overlap real situation during the training. Phantom Omni is the most frequently used for touch and palpation examinations [11]. It can generate the output force response of the radial artery pulse waveform in different cases. The pulse simulation generates pulse waveforms by the mathematical model of Gaussian functions. The simulation is useful and effective diagnostic training for the nursing students.

\section{Methods}

\subsection{Implementation of the haptic force feedback}

The haptic can respond the force feedback by the implementation method of radial artery pulse force that includes a mathematical modeling of the pulse waveform. Our previous study [12] reported the mathematical modeling of the pulse waveform using three Sine functions in the virtual pulse simulation. But the Sine function is less flexible than the Gaussian function in parameter configuration of the amplitude of each wave peaks. de Sá Ferreira et al, [13] modified the pulse waveform of hypertensive subjects and analyzed into a pulse waveform pattern using Gaussian functions. We used four graphs of the Gaussian function to generate the radial artery pulse waveform for increasing realistic pulses and close to the real pattern.

The pulse waveform pattern $p(t)$ was separated into two phases of waves traveling as progressive $f(t)$ and regressive $g(t)$ waves from the heart to the organs in Equation (1). In each phase, the wave was conducted by the time domain superimposed of two Gaussian functions as an overlap of two sine waves at different frequencies (See in Figure 1). The progressive waveform $\mathrm{f}(\mathrm{t})$ could be described as the summation of two pressure waves in Equation (2). And the regressive waveform $g(t)$ was modeled by the time domain pressure of phase superposition in Equation (3).

$$
\begin{gathered}
p(t)=f(t)+g(t) \\
f(t)=P_{1} \cdot e^{\left[\left(t-t_{P 1}\right)^{2} / \sigma_{P 1}^{2}\right]}+P_{2} \cdot e^{\left[\left(t-t_{P 2}\right)^{2} / \sigma_{P 2}^{2}\right]} \\
g(t)=R_{1} \cdot e^{\left[\left(t-t_{R 1}\right)^{2} / \sigma_{R 1}^{2}\right]}+R_{2} \cdot e^{\left[\left(t-t_{R 2}\right)^{2} / \sigma_{R 2}^{2}\right]}
\end{gathered}
$$

Where $P_{i}$ is an amplitude of each progressive waves, $R_{i}$ is an amplitude of each regressive waves, tpi means the area of systolic wave peaks, tRi is the area of diastolic wave peaks, $\sigma_{P i}^{2}$ and $\sigma_{R i}^{2}$ represent the duration of the forward and backward waves and , $\mathrm{i}$ is a number of waves ( 1 and 2). 


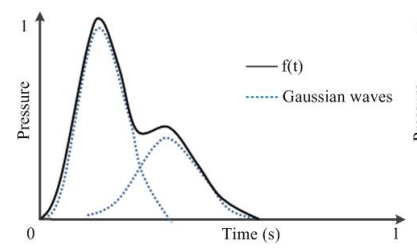

(a)

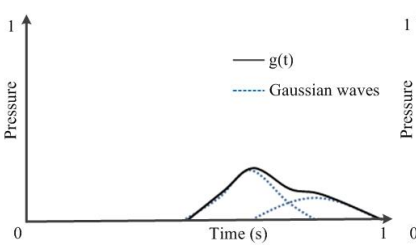

(b)

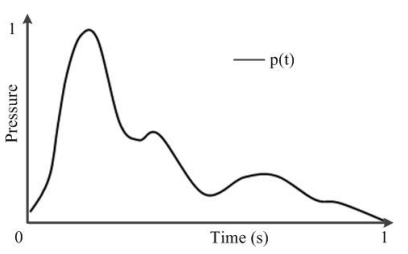

(c)

Fig. 1. The pulse waveform pattern: (a) the progressive waveform, (b) the regressive waveform and (c) The pulse waveform [13]

\subsection{Characteristics of the radial artery pulse waveform}

In the virtual pulse simulation, the pulse waveform types consist of normal pulse and abnormalities of the radial artery pulse (i.e., small and weak pulses, large and bounding pulses, bisferiens pulses and pulsus alternans) as shown in Figure 2.

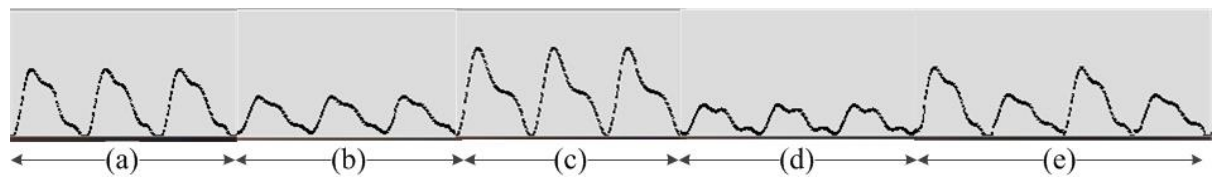

Fig. 2. The pulse waveform types

During the training, the simulation can set pulse parameters e.g. pulse rate 40-160 beats per minute (BMP), pulse pressure (volume or force) $0-1$ Newton (N.) and selected pulse type. The amplitude of wave peaks depends on estimating the parameters of Gaussian functions.

\subsection{Measuring tool}

To check the accuracy of the haptic force feedback (pulse rate generation), the output from wearable smart band device (Xiaomi MiBand 2) with optical sensors that read the mannequin's wrist (instead of the fingers) tracked the generating of pulse rate and was used to compare with the output from the pulse simulation setup. The pulse rate tracking of wearable smart band was accurate that compared with the setting up parameters of the simulation. The setups are shown in Figure 3. 


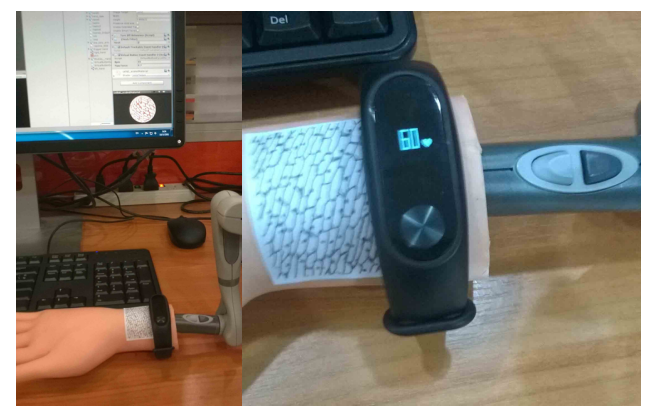

Fig. 3. The evaluation method of pulse rate generation

To evaluate this learning tool, 19 first-year students from School of Nursing were participated on voluntary basis. The population is $17 \%$ of all the first-year nursing students. All of the participants were assigned to use the pulse simulation to measure the pulse and learn about the types of pulse waveform. The characteristics of the participating nursing student are shown in Table 1.

Table 1. The characteristics of the participating nursing student

\begin{tabular}{|c|c|c|}
\hline & $\mathbf{n}$ & $\%$ \\
\hline $\begin{array}{ll}\text { Gender } & \\
& \text { Male } \\
& \text { Female }\end{array}$ & $\begin{array}{c}1 \\
18\end{array}$ & $\begin{array}{c}5.26 \\
94.74 \\
\end{array}$ \\
\hline Age (average) & 19 & \\
\hline $\begin{array}{l}\text { Experience of pulse measurement } \\
<10 \text { times/week }\end{array}$ & 19 & 100 \\
\hline $\begin{array}{l}\text { Experience of augmented reality in medical training } \\
\text { Yes } \\
\text { No }\end{array}$ & $\begin{array}{c}1 \\
18\end{array}$ & $\begin{array}{c}5.26 \\
94.74\end{array}$ \\
\hline $\begin{array}{l}\text { Experience of using the haptic device in medical training } \\
\text { Yes } \\
\text { No }\end{array}$ & $\begin{array}{c}1 \\
18\end{array}$ & $\begin{array}{c}5.26 \\
94.74\end{array}$ \\
\hline
\end{tabular}

Note. $\mathrm{n}=$ a number of student, $\%=$ a percentage of population.

\section{$4 \quad$ Experimental Setup}

\subsection{Hardware and Software}

The pulse simulation consisted of both hardware and software. The hardware parts comprise a workstation, Phantom Omni haptic device, camera, silicone mannequin hand and wearable smart band. The workstation is a computer which is connected to the camera and Phantom Omni to process input images and output forces. In order to get pulse forces, the AR marker is stuck on silicone mannequin hand that connected to Phantom Omni. The wearable smart band is used for validating the output forces (See in Figure 4). 
While the software is used for the connection between the haptic device (Phantom Omni) and the computer (workstation) such as SensAble PDD, OpenHaptics Toolkit and HD API Wrapper which run on Windows 7 64-bit. Another software that were used for the implementation of virtual pulse simulation are C\# language and Unity $3 \mathrm{~d}$ program for the programming of pulse forces and waveforms, Autodesk Maya for the building of 3D simulation and Vuforia SDK for the AR marker tracking.

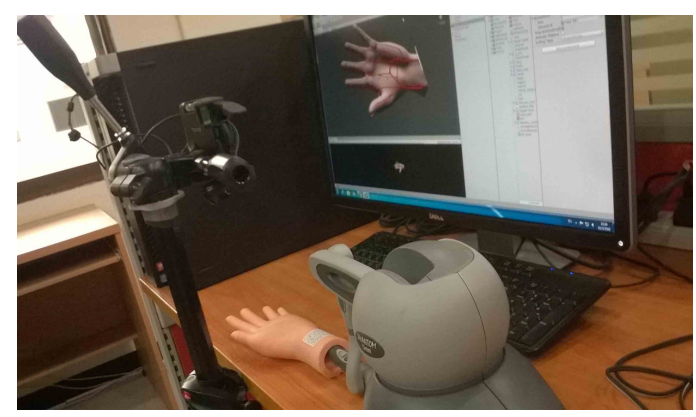

Fig. 4. The experimental setup of the training

\subsection{Experiments}

Five experiments of the pulse simulation consist of 1) the traditional method for pulse measurement, 2) the measurement of pulse volume with the simulation, 3) the measurement of the virtual pulse simulation, 4) the identification of virtual pulse between normal and abnormal cases and 5) the diagnosis of pulses in various situations. Details are shown in Figure 5 (a) - (d).

a) Experiment 1: In traditional pulse measurement method, the nursing students will measure the volunteer's pulse by using the student's fingers for 60 seconds to measure the pulse rate of the volunteer's wrist. While the pulse of the other hand of the volunteer was measured by the wearable smart band in Figure 5 (a). The aim of the experiment is to compare the accuracy of the pulse measurement between traditional method and the wearable device.

b) Experiment 2: The students selected the pulse volume (between 0 to $1 \mathrm{~N}$.) of the simulation which is suitable and close to the real pulse. After that, the selected volume was used as a pulse force in the pulse simulation during the training.

c) Experiment 3: This experiment is similar to the experiment 1 but the students practiced in the pulse measurement with the simulation which was set up the pulse rate and pulse volume of a normal pulse to compare the accuracy of virtual pulse simulation measurement.

d) Experiment 4: The students selected pulses between normal and abnormal pulses and identify the characteristics of abnormal pulse type e.g. 1) choosing either large pulse and normal pulse and 2) choosing either normal pulse or weak pulse.

e) Experiment 5: After the students have been trained with the virtual pulse simulation and understand about pulse waveform types in the experiments 2, 3 and 4, they will 
be asked to identify radial artery pulse waveforms in a different pattern of the pulse types which consist of normal, small and weak, large and bounding, bisferiens and pulsus alternans pulses. The aim of experiment 4 and 5 is to measure students' ability on different pulse type identification.

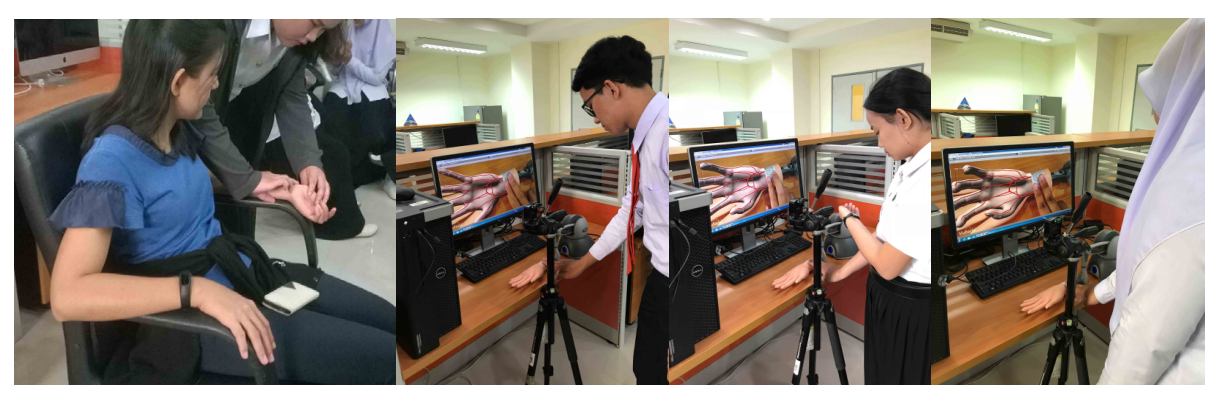

(a)

(b)

(c)

(d)

Fig. 5. The experiments: (a) traditional pulse measurement and (b) - (d) the training of pulse simulation

\subsection{Satisfaction evaluation}

The following step is a satisfaction survey of the pulse simulation. The students, who gone through the series of experiments, were asked to answer the questionnaires which consist of the general information, user satisfaction, and suggestion. Details of the satisfaction survey covered 18 items which were divided into 7 groups of questions: realistic feeling, animation rendering, usage, utilization, pulse waveform rendering, understanding before and after training. Each question has 5 points scale $(1=$ strongly disagree, to $5=$ strongly agree).

\section{$5 \quad$ Results and Discussions}

Display of the virtual 3D model as shown in Figure 6. Summary of the five experiments in the virtual pulse simulation training as shown in Table 2-4.

From the results of experiment 1, an average pulse rate that was measured by the wearable smart band is $71 \mathrm{BPM}$, while an average of the traditional pulse measurement method is 70 BPM which is $1.41 \%$ of an average value error in the pulse measurement and 4.37 of an average standard deviation score. 


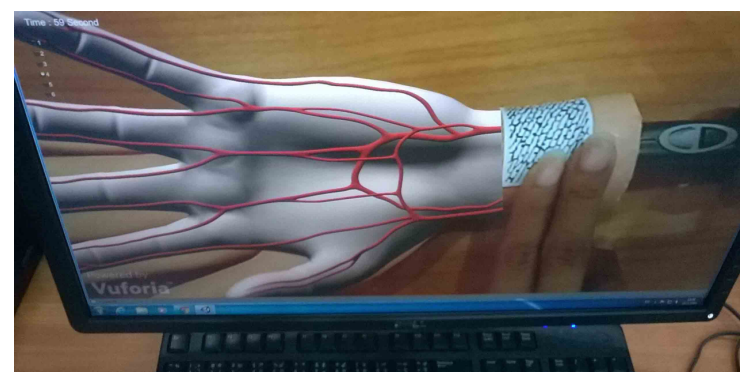

Fig. 6. The virtual display of the pulse simulation

Table 2. The results of experiment 1 and 3

\begin{tabular}{|c|c|c|c|c|c|c|}
\hline \multirow{2}{*}{ Student No. } & \multicolumn{3}{|c|}{$\begin{array}{c}\text { Experiment 1: } \\
\text { Traditional pulse measurement }\end{array}$} & \multicolumn{3}{|c|}{$\begin{array}{l}\text { Experiment 3: } \\
\text { Virtual simulation pulse measurement }\end{array}$} \\
\hline & $\begin{array}{l}\text { Volunteer's } \\
\text { pulse rate }\end{array}$ & Measuring & $\%$ & Simulated pulse rate & Measuring & $\%$ \\
\hline 1 & 70 & 72 & 2.9 & 71 & 74 & 4.2 \\
\hline 2 & 69 & 76 & 10.1 & 71 & 72 & 1.4 \\
\hline 3 & 70 & 72 & 2.9 & 71 & 68 & 4.2 \\
\hline 4 & 71 & 72 & 1.4 & 71 & 57 & 19.7 \\
\hline 5 & 72 & 74 & 2.8 & 71 & 79 & 11.2 \\
\hline 6 & 71 & 69 & 2.8 & 71 & 79 & 11.2 \\
\hline 7 & 74 & 66 & 10.8 & 71 & 72 & 1.4 \\
\hline 8 & 73 & 73 & 0.0 & 71 & 78 & 9.9 \\
\hline 9 & 70 & 65 & 7.1 & 71 & 77 & 8.5 \\
\hline 10 & 71 & 71 & 0.0 & 71 & 70 & 1.4 \\
\hline 11 & 68 & 67 & 1.5 & 71 & 72 & 1.4 \\
\hline 12 & 68 & 70 & 2.9 & 71 & 70 & 1.4 \\
\hline 13 & 71 & 72 & 1.4 & 71 & 75 & 5.6 \\
\hline 14 & 76 & 80 & 5.3 & 71 & 70 & 1.4 \\
\hline 15 & 71 & 70 & 1.4 & 71 & 68 & 4.2 \\
\hline 16 & 72 & 72 & 0.0 & 71 & 73 & 2.8 \\
\hline 17 & 73 & 71 & 2.7 & 71 & 67 & 5.6 \\
\hline 18 & 70 & 63 & 10.0 & 71 & 71 & 0.0 \\
\hline 19 & 70 & 62 & 11.4 & 71 & 70 & 1.4 \\
\hline
\end{tabular}

Note. Measuring = nursing student's measuring the pulse, $\%=$ a percentage of absolute error.

In the experiment 3, the pulse rate parameter of the virtual pulse simulation was set at $71 \mathrm{BPM}$ and an average of pulses measured by the students is $71 \mathrm{BPM}$ which is $100 \%$ accuracy and the average standard deviation score is 5.11 as shown the detail of experiment 1 and 3 in Table 2 . Table 3 shows the details of pulse force selecting. The students selected the force value $0.45,0.50$, and $0.55 \mathrm{~N}$. that is $0.5 \mathrm{~N}$. of the average pulse volume in the experiment 2 and used this value to set the pulse pressure parameter. During the simulation of experiment 2 and 3, the students also learn about the normal pulse waveform. 
Table 3. The results of experiment 2

\begin{tabular}{|c|c|c|}
\hline Experiment & $\mathbf{n}$ & $\mathbf{\%}$ \\
\hline Experiment 2: Selecting of pulse volume in different pulse force & & \\
1. $0.45 \mathrm{~N}$. & 3 & 15.79 \\
2. $0.50 \mathrm{~N}$. & 13 & 68.42 \\
3. $0.55 \mathrm{~N}$. & 3 & 15.79 \\
\hline
\end{tabular}

Note. $\mathrm{n}=$ a number of student, $\%=$ a percentage of population.

Table 4. The results of experiment 4 and 5

\begin{tabular}{|c|c|c|}
\hline \multicolumn{1}{|c|}{ Experiment } & $\mathbf{n}$ & $\mathbf{\%}$ \\
\hline Experiment 4: Choosing between normal and abnormal pulses & & \\
1. Normal and bisferiens pulses & 12 & 70.59 \\
2. Normal and large pulses & 17 & 89.47 \\
3. Normal and pulsus alternans pulses & 12 & 70.59 \\
4. Normal and weak pulses & 17 & 89.47 \\
\hline Experiment 5: Identification of various pulse types & 1 & \\
1. Bisferiens pulse & 16 & 8.26 \\
2. Large and bounding pulse & 7 & 36.84 \\
3. Pulsus alternans pulse & 4 & 21.05 \\
\hline
\end{tabular}

Note. $\mathrm{n}=$ a number of correct answer's student, $\%=$ a percentage of accuracy.

The results of choosing between the normal and abnormal pulse waveforms in the experiment 4 are $89.47 \%$ accuracy for the large and weak pulses and $70.59 \%$ accuracy for the bisferiens and pulsus alternans pulses diagnosis. In the experiment 5 , the accuracy of pulse type identifications (i.e., large and bounding, pulsus alternans, small and weak and bisferiens pulses) were $84.21 \%, 36.84 \%, 21.05 \%$ and $5.26 \%$, respectively (See in Table 4). The accuracy of some results in experiment 5 is low such as bisferiens pulses, because the simulation generates the different pulse types during the examination of pulse measurement. The students cannot compare the pulse that they feel with the normal pulse, so they must be trained on the characteristic of the pulse waveform types with the pulse simulation in order to get increase the accuracy of the pulse type identification.

The students can measure the pulse with high accuracy in the simulation as same as the traditional measurement. Also pulse characteristics identification between the normal and abnormal are high accuracy but the identification accuracy of mixed pulse types are less accuracy. Some nursing students can describe the characteristic of various pulses, so the virtual pulse simulation can be used as tool to train the students to have better understanding on the pulse waveform pattern and improving touch skill which is beneficial to an accuracy in early diagnosis.

The results of the user satisfaction survey are summarized in Figure 7. The average score of 7 question groups is $3.79,3.84,4.00,4.28,4.11,3.84$ and 4.21 point respectively. 


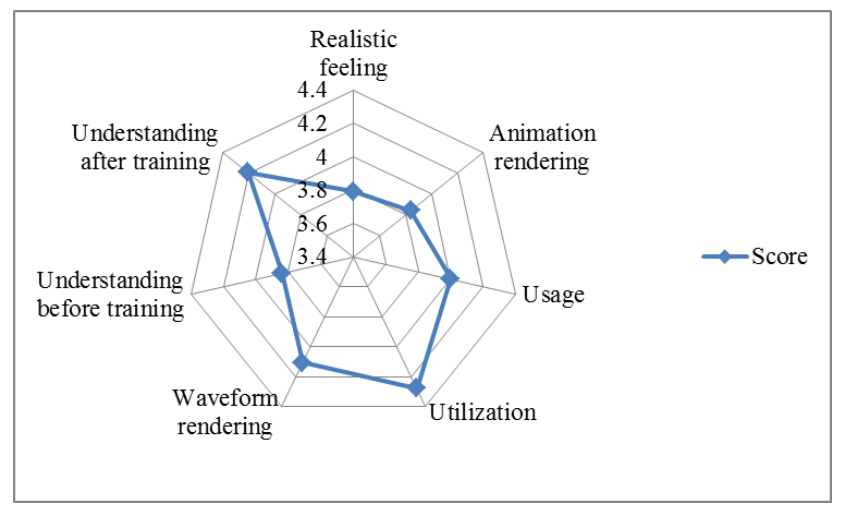

Fig. 7. The user satisfaction evaluation results

The experimental results of the virtual pulse simulation shown that the pulse measurement from 19 of first-year nursing students using the simulation are closed to the real pulse. The virtual pulse simulation can be used to check whether the students' answer of the pulse measuring correct or not. In the simulation, the pulse rate, pulse pressure, and pulse types were controlled by assigning different values to parameters and was generated the comparative pulse force for some diseases such as hypertension or heart failure which not be found in a normal case. In addition, the simulation can be effectively used for pulse diagnosis training studied in different situations.

From the satisfaction survey result, the lowest score is the realistic feeling by the reason of discontinuous pulses that were dependent on light and shadow effects when the camera detects the AR marker. However, the understanding after training and utilization are a high average score of the user satisfaction survey. The students noticed that the mannequin hand was shaken when the haptic device generated the pulse and made the result different from the real pulses.

\section{Conclusion}

In conclusion, we have proposed the development of the virtual pulse simulation using AR and haptic device for pulse diagnosis studies under various situations. The generating of radial artery pulse force and waves were modeled by Gaussian functions which created the pulse waveform types in the different cases of the disease. The accuracy of the pulse rate of this simulation was compared with the wearable smart band. This simulation supported the students' sense of touch in measuring the virtual pulse using their finger, just like in the traditional method. Results from the experiment of pulse measurement in the training system were evaluated by the nursing students in terms of the accuracy and usability satisfaction. The method of simulation-based training helps the students to gain skills and knowledge of the pulse measurement. This techniques, functions, and tools will provide a better improvement of mechanisms, challenges and future possibilities in medical education. 


\section{Acknowledgment}

The research funding is being supported by Rajamangala University of Technology Srivijaya.

\section{$7 \quad$ References}

[1] Varalakshmi, B. D., Thriveni, J., Venugopal, K. R., \& Patnaik, L. M. (2012). Haptics: state of the art survey. International Journal of Computer Science Issues, 9(5), pp. 234-244. https://www.researchgate.net/publication/266618819

[2] Liu, L. M., Li, W., \& Dai, J. J. (2017, August). Haptic technology and its application in education and learning. In Ubi-media Computing and Workshops (Ubi-Media), $201710^{\text {th }}$ International Conference on IEEE, pp. 1-6. https://doi.org/10.1109/UMEDIA.2017.8074138

[3] Ullrich, S., Mendoza, J., Ntouba, A., Rossaint, R., \& Kuhlen, T. (2008). Haptic pulse simulation for virtual palpation. In Bildverarbeitung für die Medizin 2008, Springer, Berlin, Heidelberg, pp. 187-191. https://doi.org/10.1007/978-3-540-78640-5_38

[4] Ullrich, S., \& Kuhlen, T. (2012). Haptic palpation for medical simulation in virtual environments. IEEE Transactions on Visualization and Computer Graphics, 18(4), pp. 617-625. https://doi.org/10.1109/TVCG.2012.46

[5] Coles, T., John, N. W., Gould, D. A., \& Caldwell, D. G. (2009, February). Haptic palpation for the femoral pulse in virtual interventional radiology. In Advances in Computer-Human Interactions, 2009. ACHI'09. Second International Conferences on IEEE, pp. 193-198. pp. 193-198. https://doi.org/10.1109/ACHI.2009.61

[6] LeClair, A., \& Wolfer, J. (2015, June). Haptic representation of aortic pressure waveforms using synthetic ECG derived time intervals. In Experiment@ International Conference (exp. at'15), $20153^{\text {rd }}$ on IEEE, pp. 310-315. https://doi.org/10.1109/EXPAT.2015.7463286

[7] Liu, C., Zheng, D., Murray, A., \& Liu, C. (2013). Modeling carotid and radial artery pulse pressure waveforms by curve fitting with Gaussian functions. Biomedical Signal Processing and Control, 8(5), pp. 449-454. https://doi.org/10.1016/j.bspc.2013.01.003

[8] Liu, C., Zhao, L., \& Liu, C. (2014). Effects of blood pressure and sex on the change of wave reflection: evidence from Gaussian fitting method for radial artery pressure waveform. PloS one, 9(11), e112895. https://doi.org/10.1371/journal.pone.0112895

[9] Zhu, J., Zhu, C., \& Sun, Y. (2016). Theoretical derivation and experimental statistical analysis on radial vascular mechanical modeling within the effects of hypertension. International Journal of Innovative Computing Information and Control, 12(4), pp. 1165-1178.

[10] Fan, Z., Zhang, G., \& Liao, S. (2011). Pulse wave analysis. In Advanced Biomedical Engineering, InTech. https://doi.org/10.5772/22600

[11] de Lara Ribeiro, M., \& Nunes, F. L. (2014, May). Systematically Reviewing Techniques and Devices Used in Palpation Simulation with Haptic Feedback. In Virtual and Augmented Reality (SVR), 2014 XVI Symposium on IEEE, pp. 258-267. https://doi.org/10.1109/SVR.2014.58

[12] Kandee, M., Boonbrahm, P., \& Tantayotai, V. (2017, July). Modeling realistic virtual pulse of radial artery pressure waveform using haptic interface. In Computer Science and Software Engineering (JCSSE), $201714^{\text {th }}$ International Joint Conference on IEEE, pp. 1-5. https://doi.org/10.1109/JCSSE.2017.8025954

[13] de Sá Ferreira, A., Barbosa Filho, J., Cordovil, I., \& de Souza, M. N. (2012). Noninvasive pressure pulse waveform analysis of flow-mediated vasodilation evoked by post-occlusive reactive hyperemia maneuver. Biomedical Signal Processing and Control, 7(6), pp. 616-621. https://doi.org/10.1016/j.bspc.2012.03.001 


\section{Authors}

Moragot Kandee a Ph.D. candidate in Management Information Technology Program at Walailak University, Thailand, under the supervision of Assoc.Prof.Dr. Poonpong Boonbrahm and Asst.Prof.Dr. Valla Tantayotai. Her research is in the areas of realistic modeling of virtual touch in medical training.

Dr. Poonpong Boonbrahm is an Associate Professor and Dean of School of Informatics, Walailak University, Thailand. His current research of interests included Human Computer Interactions (HCI), and Applications of Augmented Reality and Virtual Reality technology in various areas such as Education and Medical Training.

Dr. Valla Tantayotai is an Assistant Professor and former Dean of School of Nursing, Walailak University, Thailand. Her current research focuses on the risk factor assessment and intervention for diabetes prevention.

Article submitted 31 July 2018. Resubmitted 29 August 2018. Final acceptance 02 September 2018. Final version published as submitted by the authors. 